Informe especial

\title{
Vigilancia de la lepra en situaciones de baja prevalencia
}

\author{
C. Edilberto González Ochoa ${ }^{1}$ y Alfredo Abreu ${ }^{2}$
}

RESUMEN La mayoría de los países latinoamericanos han logrado reducir la prevalencia de la lepra a menos de 1 caso por cada 10000 habitantes. En estos países, la etapa siguiente es eliminar la enfermedad en el ámbito subnacional, en los territorios que tienen tasas mayores de 1 caso por 10 000. Elementos como la transición demográfica, la existencia de áreas con elevada transmisión y la necesidad de emplear indicadores más sensibles obligan a modificar las estrategias básicas, fortalecer los sistemas de vigilancia y reorientar recursos según sea necesario. Es importante renovar el empleo de tácticas como la identificación de las áreas críticas, las intervenciones diferenciadas, la concentración de indicadores y la conjugación de la vigilancia pasiva y activa. Esto puede formularse rediseñando los sistemas de vigilancia para integrar los componentes clínico, de laboratorio, de investigación epidemiológica y de suministros. Los resultados del proceso deben aportar un conjunto mínimo de indicadores que permitan monitorear y evaluar la efectividad y la eficiencia del plan de acción para la etapa posteliminación.

Palabras clave Lepra, sistema de vigilancia.

En la práctica se ha demostrado que no era demasiado optimista la antigua aspiración de eliminar la lepra como problema de salud pública. La carga mundial de la enfermedad se ha reducido de 10,5 millones de casos en 1985 a 1,15 millones a comienzos de 1997 (1). En el 2000 muchos países se aproximan a la prevalencia de 1 caso por 10000 habitantes en tanto que otros ya la han alcanzado $(2,3)$. En la Región de las Américas, en 1996 la prevalencia por 10000 habitantes era de 0,94 en la Argentina; 0,94 en Bolivia; 0,45 en

1 Toda la correspondencia debe enviarse a este autor a la siguiente dirección: Instituto Pedro Kourí, Subdirección de Epidemiología, Apartado 601, Playa, La Habana. Correo electrónico: ochoa@ipk.sld.cu

2 Cuba, Ministerio de Salud Pública, Grupo Nacional de Dermatología, Hospital Docente Comandante Fajardo, La Habana.
Costa Rica; 0,56 en Cuba; 0,49 en el Ecuador; 0,05 en El Salvador; 0,07 en Guatemala; 0,16 en Haití; 0,15 en Honduras; 0,38 en México; 0,45 en Nicaragua; 0,4 en Panamá; 0,11 en el Perú; 0,61 en la República Dominicana, y 0,18 en el Uruguay (3).

Para eliminar la lepra de las Américas, es importante comprender la epidemiología de esa enfermedad en los países que ya han logrado expulsarla de su territorio. Como parte de ese proceso, ha sido necesario ubicar precisamente los estados, las provincias, las zonas geográficas, los focos o áreas críticas que requieren tratamiento especial, ya sea porque aún no han llegado a eliminar del todo la enfermedad o porque es preciso consolidar su eliminación (4). Las acciones de nivel subnacional requieren fortalecer los sistemas de vigilancia junto con una renovación del compromiso de las autoridades nacionales y reorientar los servicios, así como la asignación de los recursos. Este último aspecto adquiere mucha importancia cuando hay dudas o reticencias en cuanto a asignar prioridad a un problema de salud que se considera ya eliminado frente a otros prevalecientes en un panorama de recursos limitados.

No cabe duda de que la lepra continúa teniendo tanta importancia y prioridad como la que recibió al inicio del plan de acción para su eliminación a partir de la Resolución WHA 44.9 aprobada en mayo de 1991 por la Asamblea Mundial de la Salud (1). Se incrementó así el compromiso político en relación con la lepra y se intensificaron las actividades para su eliminación en los países endémicos. La resolución instaba a los Estados Miembros 
con zonas endémicas de la enfermedad a aumentar o mantener el compromiso político y dar alta prioridad a alcanzar la eliminación mundial antes del año 2000; a fortalecer las actividades de la gerencia de los programas de control de esta enfermedad y a mejorar la capacitación en este sentido, tanto en los estudiantes de medicina como en los de enfermería; a alcanzar el más alto nivel de cobertura de la poliquimioterapia (PQT) y de adherencia al tratamiento; a reforzar las actividades de localización de casos apoyadas en la participación comunitaria y en la capacitación de los agentes de salud; a integrar las actividades de lucha contra la enfermedad a los servicios generales de salud y a apoyar social y económicamente a estos enfermos; a mejorar los sistemas de información nacionales de manera que faciliten la vigilancia y evaluación de las actividades de eliminación y, por último, a coordinar los recursos técnicos y financieros obtenidos a través de los organismos internacionales y no gubernamentales para su utilización óptima.

En los países con baja prevalencia, la disminución de la lepra en el nivel subnacional significa reducir de forma importante la frecuencia de las discapacidades físicas y los sufrimientos que produce. En muchos países, las discapacidades de grado 2 pueden afectar, en promedio, a entre 5 y $10 \%$ del total de los enfermos detectados. En uno de sus documentos, la OMS indica que eliminar la lepra como problema de salud pública no significaría terminar con la enfermedad. Seguiría haciendo falta una gran cantidad de trabajo para prevenir discapacidades, rehabilitar a los pacientes y mantener las técnicas de diagnóstico y tratamiento para el pequeño número de casos nuevos que continuarían apareciendo (5).

Las características de la interacción biológica entre el hombre y Mycobacterium leprae, las posibilidades reales de disponer de una tecnología eficaz y apropiada (poliquimioterapia con medicamentos eficaces y su mejor organización mediante el tratamiento supervisado) y la disponibilidad de instalaciones, personal capacitado $\mathrm{y}$ recursos financieros, hacen que la lepra sea una de las pocas enfermedades transmisibles susceptibles de ser eliminadas, aun en la era de la pandemia del sida. Se trata de un problema ancestral, que presta nueva validez a los argumentos de la citada Resolución WHA 44.9 a favor de obtener el compromiso de cada país en un nuevo empeño de eliminar la lepra. Este artículo pretende presentar una aproximación general al diseño de un sistema de vigilancia de la lepra apropiado para el período de posteliminación o eliminación subnacional de la enfermedad.

\section{ESTRATEGIAS BÁSICAS Y TÁCTICAS RENOVADAS}

Según señala Turchi, ${ }^{3}$ la eliminación de la lepra en América Latina se relaciona con la transición demográfica, es decir, con el envejecimiento de la población y la urbanización periférica de territorios grandes y medianos; la capacidad de ajustar las tácticas nacionales a las condiciones cambiantes y las diferencias entre territorios subnacionales, así como la utilización de indicadores más sensibles y dinámicos para evaluar las estrategias fundamentales del plan de acción.

Además de estrategias tan esenciales como la detección temprana de casos contagiosos, la eliminación de fuentes de transmisión mediante PQT y la cobertura masiva de acciones descentralizadas e integradas dentro de los servicios generales de salud, hay que aplicar enfoques tácticos para identificar los focos o áreas críticas de transmisión, intervenciones diferenciadas e indicadores sensibles (4). También es preciso considerar cómo conjugar la vigilancia de la población

\footnotetext{
3 Turchi CM. "Estratégia para vigilância epidemiológica em áreas urbanas sob eliminação da hanseníase no contexto dos países latinoamericanos". Informe de consultoría presentado en la Reunión Técnica OPS/OMS de Consulta sobre eliminación de la lepra como problema de salud pública a nivel subnacional/posteliminación de la lepra. La Habana, 1-3 de abril de 1998.
}

general con la vigilancia prioritaria de la población de alto riesgo.

\section{DISEÑO DE UN SISTEMA DE VIGILANCIA EPIDEMIOLÓGICA}

En la etapa de posteliminación o eliminación subnacional de la lepra, cabe considerar no tanto diseñar como rediseñar el sistema de vigilancia epidemiológica de la enfermedad (figuras 1 y 2). Esta adecuación implica reformular o revisar aspectos tales como la importancia otorgada a los cambios detectados en relación con el territorio y el momento; la claridad y factibilidad de los objetivos del sistema; la propiedad de las definiciones de casos; las características y vinculaciones de las acciones en función de los objetivos; las modalidades que integra el sistema y la reasignación de los recursos $(6,7)$.

Se trata de que un grupo de países con desarrollo disímil de los servicios de salud renueven sus esfuerzos por eliminar la lepra dentro de las estrategias y políticas comunes de atención primaria y sistemas locales de salud. Es obvia la necesidad de considerar distintas variantes de la vigilancia tradicional y la vigilancia alternativa en lo referente a vigilancia pasiva y activa y las modalidades de la vigilancia.

Aquí llamaremos sistemas de vigilancia tradicionales a aquellos que se fundamentan en datos procedentes de la declaración obligatoria de enfermedades y otros registros de enfermedades o eventos epidemiológicos en hospitales, laboratorios, centros laborales $\mathrm{u}$ otras instituciones de la seguridad social, así como de las estadísticas vitales. Por otra parte, los sistemas alternativos se nutren fundamentalmente de información adicional no contenida en los sistemas tradicionales para problemas de salud prioritarios y sus determinantes. La notificación es más detallada y diferenciada, y puede incluir elementos complementarios obtenidos de los denominados "procesos centinela".

Por proceso de la vigilancia centinela o simplemente proceso centinela debe entenderse un conjunto de elementos 
FIGURA 1. Vigilancia posteliminación de la lepra

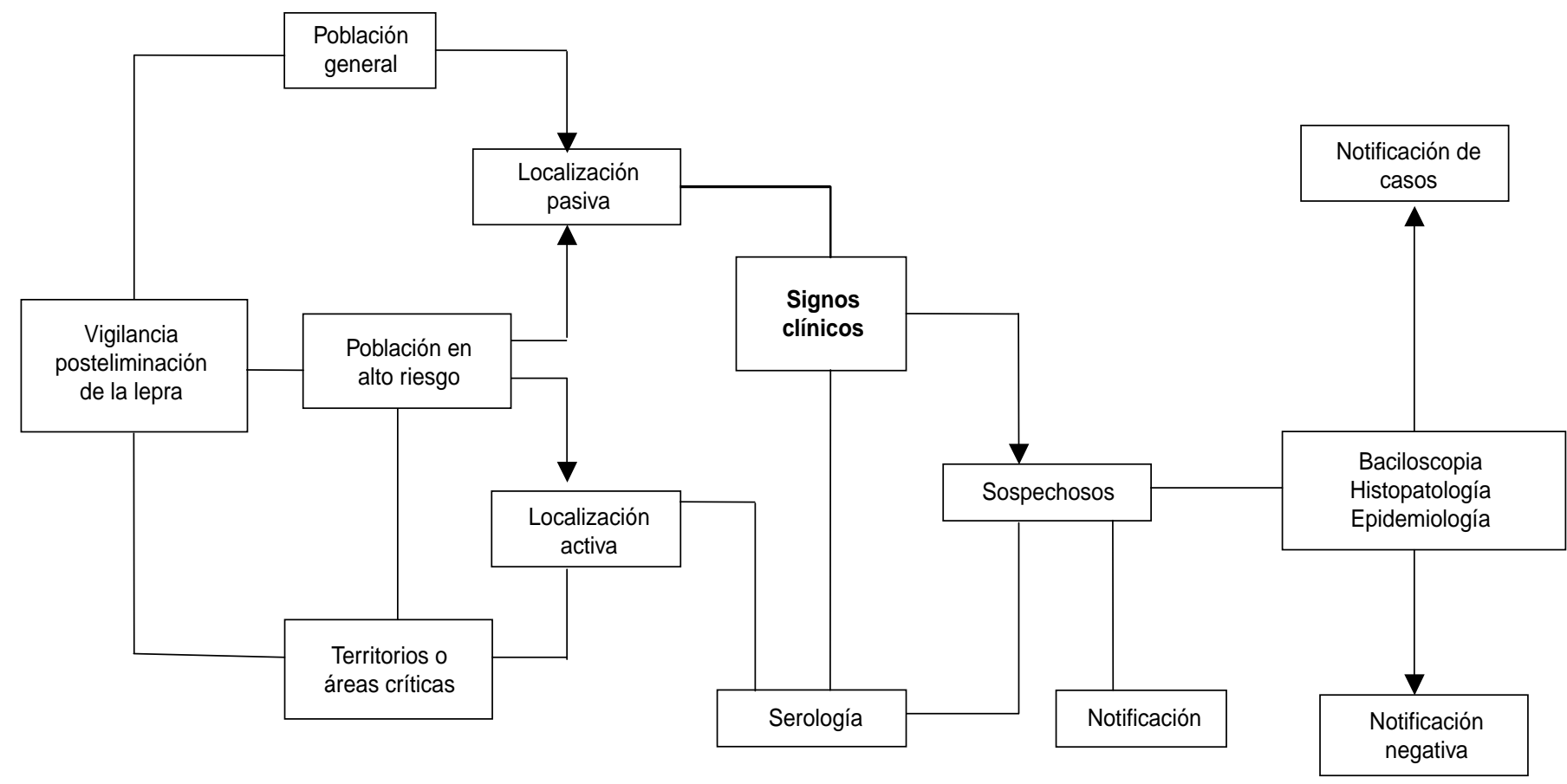

técnicos de la organización de los servicios en el mantenimiento de la vigilancia para reconocer o identificar distintos aspectos de la salud, sus determinantes y el impacto de la respuesta social para su solución. Incluye modalidades distintas, entre ellas eventos centinela, poblaciones centinela, centros centinela, sitios centinela ${ }^{4}$ y extensa aplicación de técnicas de pesquisa activa. En el período de posteliminación se suele aplicar el proceso centinela de la vigilancia alternativa o complementaria, sin perjuicio de la tradicional.

\section{Objetivos de la vigilancia en el período posteliminación}

En el período posteliminación de la lepra, el objetivo principal es obtener información pertinente sobre indica-

4 González E. Sistemas alternativos en la vigilancia de las infecciones respiratorias agudas en Cuba [tesis doctoral]. La Habana: Instituto Pedro Kourí; 1996. dores que revelen una reducción adicional de la prevalencia a menos de 1 caso por 10000 habitantes, los casos detectados en menores de 15 años y los casos detectados tardíamente en los territorios y poblaciones subnacionales donde se haya comprobado la transmisión de la enfermedad en años recientes. Los indicadores operativos de los cuales se obtiene información de cobertura, impacto y costos permiten monitorear o evaluar el progreso y el alcance del plan de acción para la eliminación subnacional. En los países de baja prevalencia es especialmente importante que se incluyan también los indicadores de notificación negativa. El silencio o ausencia de notificación se suele considerar favorablemente atendiendo al mantenimiento o incremento de las acciones de detección de tal modo que puedan verificarse objetivamente los resultados del programa. ${ }^{5}$ Un conjunto de indicadores

\footnotetext{
5 World Health Organization. WHO-recommended surveillance standards for communicable diseases. (WHO/EMG/DIS/97.1).
}

que darían cumplimiento a los objetivos principales pueden seleccionarse de la siguiente serie $(7,8)$ :

- Número, proporción y tasa de casos nuevos detectados (tasas específicas por edad y tipo de lepra).

- Número, proporción y tasa de casos nuevos diagnosticados tardíamente.

- Prevalencia de casos de lepra según los tipos clínicos y microbiológicos.

- Número y proporción de casos con discapacidades.

- Número y proporción de casos multibacilares entre los casos nuevos.

- Número y proporción de casos curados con PQT.

- Cobertura del tratamiento con PQT.

- Número y proporción de casos en tratamiento con PQT.

- Número de meses-paciente; número y proporción de paquetes tipo blíster usados.

Entre estos indicadores, debe considerarse de especial importancia cuantificar los casos nuevos detectados, por edad y tipos; los casos nuevos detecta- 
FIGURA 2. Vigilancia posteliminación de la lepra: flujo de los datos y la información

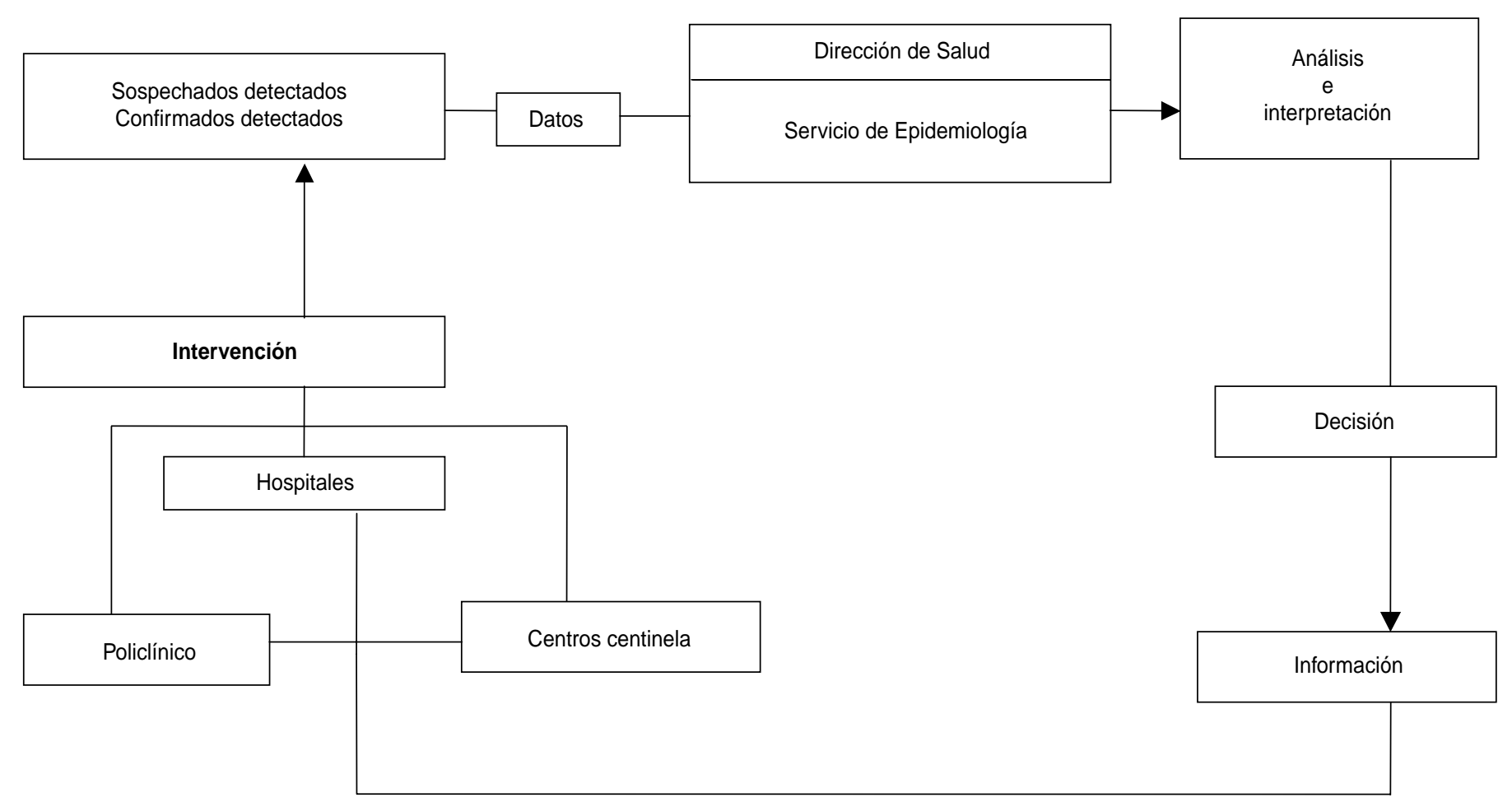

dos tardíamente; los casos tratados con PQT y los curados por ese medio; y los enfermos en tratamiento regular.

\section{Definiciones de casos}

Llevar a cabo un proceso de vigilancia eficiente en un país donde la lepra tiene baja prevalencia es una tarea crucial pero difícil. Es necesario obtener a bajo costo la información más exacta y confiable posible con el fin de detectar cualquier pequeño remanente de prevalencia oculta. Esto significa también reducir paulatinamente los casos nuevos detectados tardíamente. Deberían emplearse, por lo tanto, definiciones que permitan aumentar la sensibilidad del sistema de detección de casos sin causar una gran disminución de su especificidad.

Para aumentar la sensibilidad se podría, por ejemplo, establecer la categoría de sospecha de lepra para el tamizaje en serie, junto con un diagnóstico confirmatorio que satisfaga la definición de caso de lepra. Esta categoría de sos- pecha se ha incluido en algunas guías de vigilancia (5) y se refiere a personas que presentan uno o más de los signos siguientes:

- Áreas físicas de anestesia, hipoestesia o parestesia.

- Manchas hipocrómicas o eritematohipocrómicas, con o sin disminución de la sudación y el pelo.

- Placas eritematosas de límites nítidos con alteración de la sensibilidad.

- Uno o varios troncos nerviosos engrosados o dolorosos.

- Placas eritematosas infiltradas con el centro deprimido.

- Eritemas, infiltraciones difusas.

- Placas eritematosas difusas con bordes difusos.

- Nódulos.

- Alopecia de la cola de las cejas.

- Engrosamiento del pabellón auricular.

La corroboración de los criterios anteriores contribuye a definir un caso confirmado. Por ejemplo, caso confirmado será toda aquella persona que presente uno o más signos clínicos de sos- pecha de los enunciados anteriormente y tenga una baciloscopia positiva a M. leprae o una baciloscopia negativa con examen histopatológico compatible con la lepra, o una baciloscopia negativa con una historia epidemiológica de contacto con un caso de lepra. Las definiciones amplias de caso sospechado y de caso confirmado acordes con las características de la enfermedad pueden ser eficientes sin un número importante de positivos falsos ni tampoco de falsos negativos, es decir, con sensibilidad y especificidad razonables.

Cuando se dispone de tecnologías de tamizaje como las pruebas serológicas de ELISA para diagnóstico, tales como ultramicroELISA y UMELISAHansen (8-11), puede establecerse un proceso de tamizaje en serie o en paralelo, que permite aumentar el rendimiento y la eficiencia. Se ha recomendado $(10,11)$ que en los resultados del tamizaje se empleen las denominaciones siguientes de acuerdo con la relación de fluorescencia del suero investigado con respecto a la del suero de control positivo: negativo $<0,3$; du- 
doso 0,3-0,399; sospechado 0,4-0,499; elevado $\geq 0,500$. Debe considerarse caso nuevo detectado tardíamente todo caso nuevo notificado un año o más después del comienzo de síntomas o signos así como todo caso nuevo notificado que presente alguna discapacidad de grado 3 en el momento del diagnóstico (11). Puede definirse como área crítica una que tenga una proporción de $\geq 50 \%$ de casos nuevos detectados tardíamente o en donde se detecte algún caso nuevo en una persona menor de 15 años de edad; o una en que menos de $80 \%$ de los casos reciban PQT o donde menos de $70 \%$ de los casos reciban el tratamiento completo con PQT prescrito.

Para los efectos de un proceso centinela en la etapa posteliminación de la lepra, se proponen las siguientes definiciones:

- Población centinela: cualquier conjunto poblacional perteneciente a un territorio donde el monitoreo de la lepra permite obtener información apropiada para interpretar la situación epidemiológica y tomar decisiones al respecto.

- Evento centinela: un suceso que indica algún cambio importante en las características, tendencia o situación epidemiológica de la lepra.

- Centro o unidad centinela: centro de atención de salud que ejecuta la localización activa y pasiva de casos sospechados de lepra y casos confirmados utilizando técnicas y procedimientos sumamente sensibles y específicos, que no se emplean de ordinario en otros centros.

\section{Componentes del sistema de vigilancia epidemiológica}

Un sistema de vigilancia para cualquier problema de salud suele constar de varios componentes, o sea, conjuntos de acciones que identifican la especialidad médica pertinente o la dedicación funcional de los servicios de salud (12). A su vez, cada componente posee los tres elementos de todo sistema, es decir, la entrada, el proce- samiento y la salida de la información correspondiente. De este modo, en la vigilancia de la lepra pueden distinguirse de cuatro a cinco componentes integrados, independientemente del tipo de organización sanitaria del cual dependen, por ejemplo, del sistema nacional de salud pública, la seguridad social o una combinación de estas dos. Dichos componentes son el clínico, el de laboratorio, el de investigación epidemiológica y el de suministros. La eficiencia de la vigilancia dependerá del grado de efectividad con que funcionen todos estos componentes en los distintos centros de salud.

El sistema de vigilancia puede aplicarse por igual a territorios nacionales $\mathrm{y}$, de forma diferenciada intensa, a zonas o estratos con más de 1 caso por 10000 habitantes. A continuación se describen los componentes mencionados para zonas de baja prevalencia.

Vigilancia clínica. En este componente se realizan las actividades siguientes con objeto de identificar, describir y notificar las características de los casos sospechados y confirmados de lepra, distribuir esa información y tomar decisiones:

- Interrogar y someter a examen físico general y dermatológico a las personas que consultan espontáneamente en los centros generales periféricos de atención primaria y secundaria, para detectar signos clínicos de lepra $(1,7)$.

- Remitir a los pacientes sospechosos de lepra a un centro de referencia que cuente con los recursos para realizar los exámenesbaciloscópicos e histopatológicos pertinentes $\mathrm{o}$, en su defecto, a un dermatólogo que pueda realizar esos exámenes.

- Realizar tamizajes dermatológicos en grupos especiales de población de alto riesgo ubicados en zonas críticas, territorios o bolsones considerados focos de transmisión de la lepra, a manera de poblaciones o grupos centinela.
- Seguir el resultado de la PQT de los casos de lepra y la profilaxis de los contactos.

- Clasificar, registrar y notificar los casos sospechados y confirmados de lepra según las categorías estándar de la OMS.

- Describir la frecuencia, los patrones y la tendencia, así como el impacto de la PQT en los casos sospechados y confirmados, según edad, sexo, factores socioeconómicos, resultados baciloscópicos, tipos clínicos y territorios.

- Elaborar y diseminar informes técnicos mensuales acumulados, trimestrales acumulados y anuales.

En este componente se manejan las siguientes fuentes de datos: fichas clínicas, formularios de órdenes de laboratorio, fichas de notificación de casos sospechados y confirmados, y censos y estimaciones de la población (total y de grupos específicos).

Laboratorio. Los objetivos del componente de laboratorio consisten en identificar la presencia de $M$. leprae en frotis tomados de presuntos pacientes de lepra o de biopsias de personas con signos histopatológicos evidentes; detectar concentraciones significativas de anticuerpos anti- $M$. leprae en muestras de suero de presuntos pacientes $\mathrm{y}$, por último, describir y diseminar la información de esos resultados.

Las actividades del componente de laboratorio pueden plantearse dentro de los términos siguientes:

- Identificación del número de frotis positivos a M. leprae dentro del conjunto investigado por sospecha de lepra, incluidas las personas que han acudido a consulta de rutina y las investigadas como contactos en los focos.

- Identificación de biopsias positivas procedentes de personas con lesiones compatibles con el diagnóstico de lepra, personas que han acudido a consulta de rutina y otras detectadas mediante el tamizaje activo. 
- Identificación de muestras de suero con células de UMELISA con positividad para anticuerpos de $M$. leprae en poblaciones sometidas a tamizaje sistemático o masivo dentro de territorios de alto riesgo.

Las principales fuentes de datos de este componente son los resultados de las baciloscopias y pruebas serológicas UMELISA-Hansen.

Investigación epidemiológica. Para precisar el diagnóstico de los casos de lepra entre las personas que acuden a consulta en los centros de salud y evaluar el riesgo que representan ciertos grupos de población y territorios, la investigación epidemiológica aporta pruebas de transmisión en conglomerados de pacientes y caracteriza y valora la asociación con los factores de riesgo. También identifica los patrones de estos problemas y sus tendencias, en función de las acciones siguientes:

- Identificación de las personas que hayan tenido cualquier contacto con el caso índice y descripción de su estado de salud en relación con la lepra, poniendo énfasis en encontrar la fuente oculta de la infección (casos de prevalencia oculta).
- Seguimiento de los contactos, incluida la evaluación de su tratamiento profiláctico.

- Análisis de los datos obtenidos de las fichas epidemiológicas de los contactos de casos sospechados y confirmados de lepra, con énfasis en la evaluación de los riesgos, los patrones de transmisión y las tendencias en los últimos 5 años.

- Elaboración y diseminación de informes técnicos en boletines mensuales, trimestrales y anuales.

Las fuentes de datos son las fichas epidemiológicas y los censos y estimaciones de la población total y de grupos y territorios específicos.

Suministros. El objetivo de este componente se centra en cuantificar sistemática y continuamente los insumos que son esenciales para diagnosticar y tratar los casos e investigar a los contactos. Como ejemplo, cabe mencionar la lepromina, los reactivos para baciloscopia y las pruebas de ELISA, así como las fichas de notificación e investigación epidemiológica de los casos, y los medicamentos.

- La actividad principal consiste en contar periódicamente los materiales almacenados para saber qué can- tidades se han consumido y cuáles hay que reponer a fin de mantener un surtido apropiado de todos los productos.

Los datos se obtienen de las tarjetas o fichas que indican las cantidades máximas y mínimas de reactivos y medicamentos $\mathrm{u}$ otros insumos, $\mathrm{y}$ de bases de datos correspondientes almacenados en computadoras personales.

\section{Ejemplo del proceso centinela}

A veces se identifica como área crítica un estado o una provincia entre varios territorios, o bien dos municipios dentro de una provincia. En este caso, todos los habitantes pueden constituir la población centinela. Por contraste, pueden someterse a vigilancia áreas no críticas. Pueden seleccionarse centros o unidades de salud de esa población como centros o unidades centinela, atendiendo a criterios de factibilidad $\mathrm{u}$ otros (incluso la selección aleatoria). En los centros centinela algunos médicos, enfermeras o auxiliares de salud bien entrenados, según el caso, (o todos en los centros muy pequeños) pueden actuar como centinelas. Ellos mantienen una búsqueda activa de signos clínicos de lepra para identificar a personas con

CUADRO 1. Vigilancia de la lepra: ejemplo de alternativas para el proceso centinela

\begin{tabular}{|c|c|c|c|c|}
\hline Evento & $\begin{array}{l}\text { Unidad o centro } \\
\text { de salud }\end{array}$ & Personal & Evento centinela & Sitio \\
\hline $\begin{array}{l}\text { De territorios o áreas } \\
\text { críticas seleccionadas } \\
\text { en cada estado o país } \\
\text { de acuerdo con } \\
\text { criterios convencionales } \\
\text { o normalizados } \\
\text { - De áreas no críticas } \\
\text { que sirvan de contraste }\end{array}$ & $\begin{array}{l}-1^{\mathrm{er}} \text { nivel } \\
\cdot 2^{\circ} \text { nivel }\end{array}$ & $\begin{array}{l}\text { - Médicos generales } \\
\text { - Dermatólogos } \\
\text { - Personal de } \\
\text { enfermería } \\
\text { - Técnicos de } \\
\text { laboratorio } \\
\text { - Auxiliares sanitarios }\end{array}$ & $\begin{array}{l}\text { - Caso nuevo tardío } \\
\text { - Caso nuevo en un } \\
\text { menor de } 15 \text { años } \\
\text { - Notificación negativa }\end{array}$ & $\begin{array}{l}\text { Encuesta domiciliaria en } \\
\text { busca de: } \\
\text { - Manchas hipocrómicas } \\
\text { u otros signos } \\
\text { trazadores del } \\
\text { diagnóstico de } \\
\text { sospecha de lepra } \\
\text { - Identificación de casos } \\
\text { en tratamiento con PQT } \\
\text { - Casos curados } \\
\text { - Consumo de } \\
\text { medicamentos } \\
\text { - Realización de pruebas } \\
\text { ultramicroELISA-Hansen }\end{array}$ \\
\hline
\end{tabular}


sospecha o a casos clínicos de lepra (según el nivel de atención). Subconjuntos de la población centinela considerados en mayor riesgo pueden ser encuestados en sus hogares para valorar la cobertura de PQT en enfermos conocidos, los resultados del tratamiento, $y$ la regularidad y aplicación de pruebas serológicas como la ultramicroELISAHansen (cuadro 1 y figura 3).

\section{Coordinación intra y extrasectorial}

Para instituir una vigilancia eficiente es imprescindible que actúen en concierto las distintas instituciones y agencias que atienden la salud dentro del sistema político-sanitario de cada país. Los niveles subnacionales y centrales tienen que controlar la información que reciben sobre todos los pacientes diagnosticados en servicios estatales, de seguridad social, religiosos o privados, y velar por la calidad del diagnóstico, la notificación, el tratamiento, la rehabilitación y la educación de los casos y los contactos. Sobre todo, es obligatorio que dichas agencias convengan en cumplir las orientaciones técnicas del plan de vigilancia y el programa nacional de control de la lepra. No menos importante es la concertación con otros organismos privados e instituciones estatales, así como con las agencias de financiamiento, las organizaciones no gubernamentales y otras similares, en el empeño de obtener recursos y aplicar programas educativos sociales en las áreas críticas que requieren prioridad.

\section{Análisis de datos}

El análisis de los datos depende del nivel de complejidad de los servicios y sus recursos. Puede ser más o menos complejo, pero siempre tiene que basarse en un número mínimo de los indicadores establecidos. Incluye cuadros, mapas y gráficos por territorios (que destacan las zonas críticas) y cálculos de la prevalencia, la tasa de casos nuevos detectados tardíamente por edad y tipos, y las tasas de curación (13), tratamiento regular y cobertura.
FIGURA 3. llustración esquemática de un proceso centinela

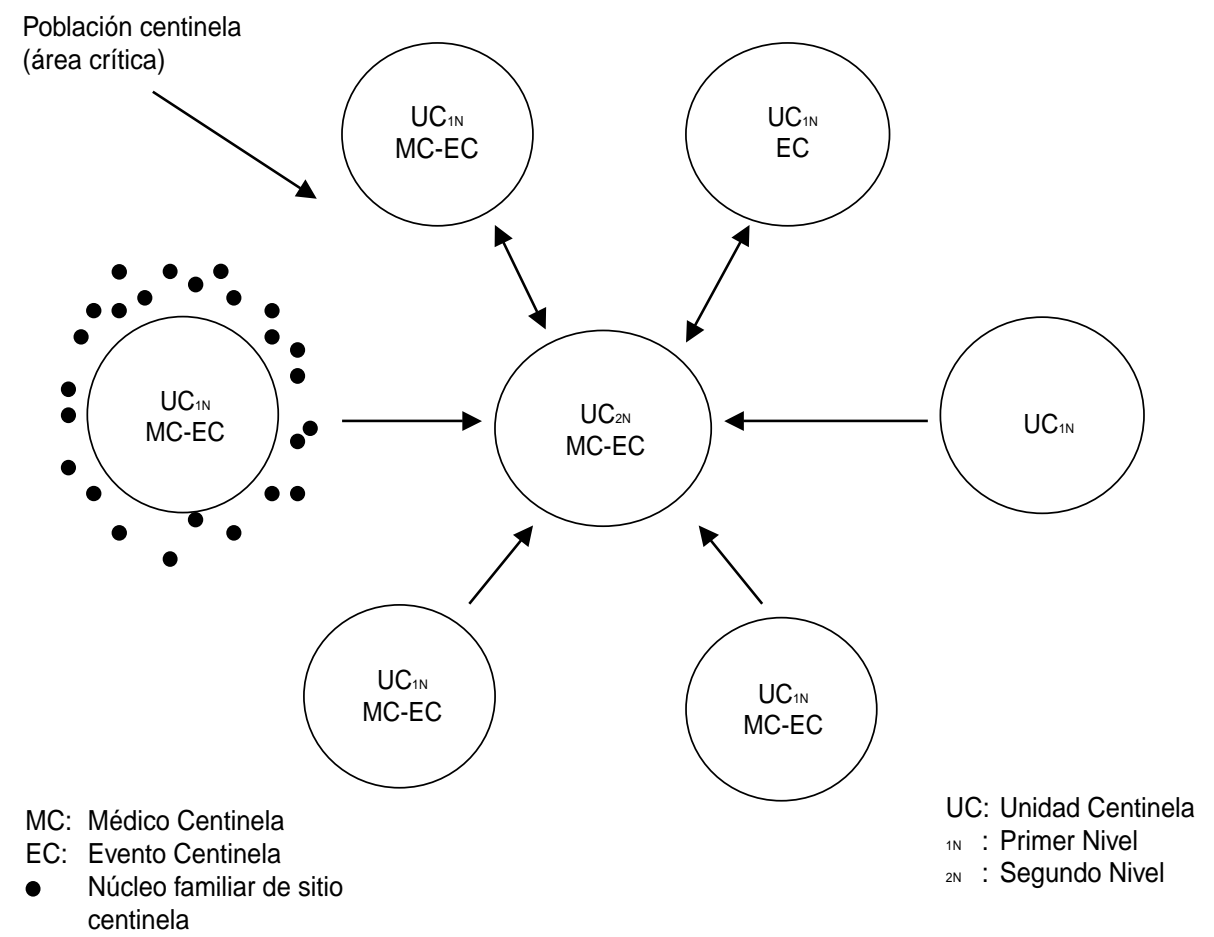

Estos datos se comparan con los valores esperados de los parámetros establecidos (cuadro 2), lo que puede realizarse por medio de paquetes de computación corrientes o elaborados para ese fin en particular.

\section{Reasignación de recursos}

Dado que el fortalecimiento de la vigilancia requiere intensificar la búsqueda activa de casos en poblaciones de riesgo y utilizar tecnologías como la prueba ultramicroELISA-Hansen, es necesario atraer nuevos recursos humanos, tecnológicos y financieros a la vigilancia. La efectividad del programa de vigilancia y control en función del costo puede ser el elemento más convincente de la conveniencia de reasignar esos recursos. En este sentido, el presupuesto general siempre debe presentarse junto con una lista de los materiales necesarios y sus precios, y un pronóstico racional del impacto esperado.

CUADRO 2. Parámetros de los indicadores para evaluar la fase posteliminación de la lepra

\begin{tabular}{ll}
\hline \multicolumn{1}{c}{ Indicador } & Valor esperado \\
\hline Tasa de casos nuevos detectados $<15$ años & 0,00 \\
Tasa de casos nuevos detectados $\geq 15$ años & $\leq 2,00$ por $10^{-5}$ \\
Prevalencia & $<1,00$ por $10^{-4}$ \\
Tasa de casos nuevos detectados tardíamente & $\leq 1,00$ por $10^{-5}$ \\
Proporción de casos con tratamiento regular & $>80 \%$ \\
Proporción de casos curados & $>90 \%$ \\
Proporción de abandono de la poliquimioterapia & $<5 \%$ \\
\hline
\end{tabular}




\section{REFERENCIAS}

1. Organización Mundial de la Salud. Comité de expertos de la OMS en lepra: séptimo informe. Ginebra: OMS; 1998. (Serie de Informes Técnicos 874).

2. Organización Panamericana de la Salud. Lepra. En: Las condiciones de salud en las Américas (vol. 1). Washington, DC: OPS; 1994: 183-185. (Publicación Científica 549).

3. Organización Panamericana de la Salud. Estrategias de la campaña de la lepra desarrollada en Brasil. Lepra al Día 1997;5:1-4.

4. Organización Panamericana de la Salud/ Organización Mundial de la Salud. Plan de Acción Regional OPS/OMS para la Consolidación de la Eliminación de la Lepra de las Américas 2000-2005. Lepra al Día 2000;8: $1-3$.

5. Organización Mundial de la Salud. Eliminación de la lepra: preguntas y respuestas. Ginebra: OMS, Programa de Acción para la Eliminación de la Lepra; 1996. (WHO/LEP/96.6).
6. Teutsch SM. Considerations in planning a surveillance system. En: Principles and practice of public health surveillance. Oxford University Press; 1994:18-30.

7. Benenson AS. Lepra. En: Manual para el control de las enfermedades transmisibles. 16. ed. Washington, DC: Organización Panamericana de la Salud; 1997: 290-294. (Publicación Científica 564).

8. Brasil, Ministério de Saúde, Fundação Nacional de Saúde. Hanseníase, vigilância epidemiológica. En: Guia de vigilância epidemiológica. Brasilia; 1994:167-176.

9. Laferte J, Abreu EG, Robaina R, Verez V. UltramicroELISA para la detección de anticuerpos IgM anti-M. leprae. Rev Inst Med Trop São Paulo 1991;33:491-495.

10. Torrella A, Solís RL, Pérez E, Medina Y, Kerguele $C$, Olaya P. UMELISA-HANSEN en el diagnóstico y seguimiento de la lepra. La Habana: Centro de Inmunoensayo; 1992.
11. Comité de Expertos de la OMS en Lepra. Sexto informe. Ginebra: Organización Mundial de la Salud; 1988. (Serie de Informes Técnicos 768).

12. González E. Vigilancia epidemiológica (en salud) y atención primaria; una alternativa cubana. La Habana: Instituto Pedro Kourí; 1998.

13. World Health Organization. Shortening and duration of treatment of multibacillary leprosy. Wkly Epidemiol Rec 1997;72(18):125-128.

Manuscrito recibido el 15 de noviembre de 1999. Aceptado para publicación, tras revisión, el 4 de diciembre

ABSTRACT The majority of the countries in Latin America have reduced the prevalence of leprosy to less than 1 case for every 10000 persons. The next step in these countries is to eliminate the disease at the regional and local level, in "pockets" that still have rates higher than 1 per 10 000. Given the demographic transition, the existence of areas with high transmission levels, and the necessity for more sensitive indicators, there is a need to change basic strategies, strengthen surveillance systems, and refocus resources. It is important to revamp efforts through such tactics as identifying priority geographical areas, customizing interventions, improving indicators, and combining passive and active surveillance. This can be done by redesigning surveillance systems to integrate the clinical, laboratory, epidemiological-research, and supply components. The results of the process should provide a minimum set of indicators that make it possible to monitor and evaluate the effectiveness and efficiency of action plans for the postelimination stage. 